population estimates. A number of statistical analyses were undertaken to calculate trends in annual notifications: (i) nonparametric Kendall tau-b correlation (reference method), (ii) a negative binomial regression model, calculating incident rate ratios (IRRs), including a step-change analysis, and (iii) a logistic regression model calculating annual reporting odds ratios for 13 common causative agents.

Results 1637 cases of OA were notified between 1991 and 2011. Most cases were notified from the Birmingham Heartlands specialist clinic and notifications made elsewhere in the West Midlands fell from 16 per million workers in 1995 to zero in 2004, with very few cases (4 per million workers) after that. A significant non-linear decrease in annual total notifications was observed over the study period (IRR $=1.056 ; 95 \%$ $\mathrm{CI}=1.012-1.102 ; \mathrm{p}=0.012)$, as was the case for most causative agents studied. However cleaning products showed a $6 \%$ increase in notifications year-on-year (IRR $=1.056$; 95\% $\mathrm{CI}=1.012-1.102 ; \mathrm{p}=0.012$; Figure 1). Although the incidence of isocyanate-related OA decreased significantly after 2005 , the proportion of annual notifications due to isocyanates relative to other agents increased year on year ( $\operatorname{tau}=0.43$; $\mathrm{p}=0.007)$.

Conclusions Falling incidence of OA between 1991 and 2011 is explained in part by under-recognition by healthcare professionals, and by reporter fatigue, though for some causative agents this may be attributed in part to better workplace controls. Isocyanates remain the most common cause of OA but reports have fallen recently along with colophony, latex and glutaraldehyde, which have almost disappeared. Conversely cleaning product related OA is increasingly recognised.

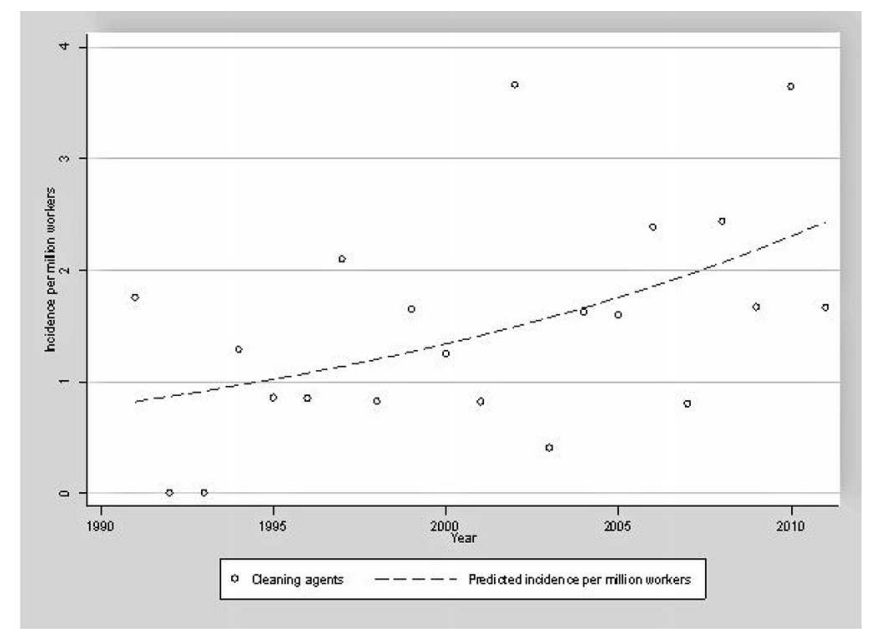

Abstract P205 Figure 1. Annual notifications of occupational asthma due to cleaning products (cases per million workers) recorded 1991-2011 in West Midlands UK, with prediction line based on negative binomial regression analysis.

\section{P206 ASBESTOS RELATED DIFFUSE PLEURAL THICKENING; LIKELIHOOD OF PROGRESSION IN THE SECONDARY CARE SETTING}

JL Hoyle, S Gudur; North Manchester General Hospital, Manchester, UK

\subsection{6/thoraxjnl-2013-204457.358}

Introduction There is a lack of recent evidence in terms of progression and prognosis in subjects with asbestos induced diffuse pleural thickening (DPT). We reviewed those with a diagnosis of DPT to look at radiological progression and prognosis.

Methods A local database search identified those registered with DPT as agreed by a Multidisciplinary Meeting (MDT) and defined by Industrial Injuries Disablement Benefit (IIDB) as including obliteration of a costophrenic angle on chest $\mathrm{x}$-ray (CXR) and mesothelioma previously ruled out. Years of followup and progression on radiology defined as a measureable increase in thickening unilaterally on CXR, or new obliteration of a costophrenic angle contralaterally. All causes of death were recorded.

Results 39 of 48 subjects registered, all male had local follow up. Radiological follow-up occurred for between 1 and 10 years, mean 3.9 yrs.

In total $16 / 39(41 \%)$ had radiological progression on CXR. 7 $(18 \%)$ died, 6/39 (15\%) from respiratory related illness. 6/39 had coexistent asbestosis.

28/39 (72\%) presented with unilateral DPT of whom 5/28 (18\%) had asbestosis on CT.

11/28 (39\%) progressed from unilateral to bilateral DPT including 3 with asbestosis, 4/28 (14\%) died, (1 with DPT and asbestosis developed mesothelioma), 1 of non-respiratory cancer, 2 died respiratory failure; however 35\% (8/23) of the unilateral DPT only cohort also progressed or died.

$5 / 11(45 \%)$ presenting with bilateral DPT progressed or died. 2 (18\%) increased thickening on CXR. 3/11 (27\%) died, 1 lung cancer (with asbestosis) and 2 respiratory failure.

Discussion Although DPT is considered benign, 41\% progressed including 39\% with unilateral DPT who developed bilateral disease. $15 \%$ had coexistent asbestosis and as expected were more likely to progress or die. $11 \%$ of those presenting with unilateral disease on CXR and over 27\% with bilateral disease died of respiratory cause over the follow-up period.

Conclusions Not withstanding the limitations of this study, the likelihood of radiological progression with a diagnosis of DPT is 2 in 5 , including those with unilateral disease. The risk of death from a respiratory cause with bilateral DPT diagnosed on CXR (27\%) is more than twice that for unilateral disease on CXR (11\%).

\section{Abstract P206 Table 1.

\begin{tabular}{|c|c|}
\hline $\begin{array}{l}\text { Unilateral DPT on CXR } \\
N=28\end{array}$ & $\begin{array}{l}\text { Bilateral DPT on CXR } \\
\mathrm{N}=11\end{array}$ \\
\hline CT & CT \\
\hline
\end{tabular}

Progression DPT only Asbestosis seen DPT only Asbestosis seen Total

\begin{tabular}{llllll} 
Stable & 15 & 2 & 6 & 0 & 23 \\
Progressed & 5 & 2 & 2 & 0 & 9 \\
Died & $3^{*}$ & 1 & 2 & 1 & 7 \\
Total & 23 & 5 & 10 & 1 & 39 \\
\hline
\end{tabular}

*1 died of non-respiratory cancer

CT-Computed Tomography of lungs

DPT-Diffuse pleural thickening

\section{P207 REPORTING OF ASBESTOS RELATED LUNG DISEASE TO HM CORONER: A RETROSPECTIVE ANALYSIS}

C Waterworth, G Hughes, B Srinivasan, BG Marshall; Southampton General Hospital, Southampton, UK

\subsection{6/thoraxjnl-2013-204457.359}

Background Asbestos related lung disease consists of a spectrum of disorders, ranging from benign pleural plaques to 
mesothelioma. In patients with a history of asbestos exposure, radiological imaging can be used to make a diagnosis. Accurate reporting of asbestos related lung disease is of vital importance to national statistics and affected individuals and their families may claim compensation from the government under the industrial injuries disablement benefit scheme. This study set out to examine the adequacy of reporting of asbestos related lung disease to HM coroner in a large teaching hospital.

Methods A retrospective case-note analysis was performed for all deaths occurring whilst under the care of respiratory physicians at a large teaching hospital from July 2012 to February 2013. Electronic patient records and radiological imaging for all of these patients was examined for any evidence of pleural plaques, pleural thickening, asbestos-related lung cancer, asbestosis or mesothelioma. A radiologist had reported all radiological imaging. Records were then cross-referenced with information held by the bereavement care office to identify whether these deaths had been referred to the coroner.

Results 115 patients were identified. Of these, 16 had radiological evidence of asbestos related lung disease; 4 on chest $\mathrm{x}$-ray and 12 on CT. 2 of these patients had biopsy confirmed mesothelioma. Both mesothelioma cases were referred to the coroner and a coroners' inquest subsequently performed. Only $60 \%$ of CT proven asbestos related disease and $30 \%$ of patients with CXR changes were referred. Of the cases not referred to the coroner 3 patients had bilateral pleural thickening on CXR and 4 patients had CT proven evidence of widespread pleural thickening.

Conclusions This study demonstrates that whilst reporting of malignant asbestos-related pleural disease to the coroner is adequate, there is room for improvement in the reporting of more benign disease. The authors propose that improved education of junior doctors may increase awareness of the importance of asbestos related lung disease, having far-reaching implications for both national statistics and those affected by these conditions.

\section{P208 TAKE UP OF INDUSTRIAL INJURIES DISABLEMENT BENEFITS AND SPIROMETRIC PATTERN ON SUBJECTS WITH ASBESTOS RELATED DIFFUSE PLEURAL THICKENING (DPT)}

S Gudur, J Hoyle; North Manchester General Hospital, Manchester, UK

\subsection{6/thoraxjnl-2013-204457.360}

Industrial injuries disablement benefit (IIDB) defines asbestos related diffuse pleural thickening (DPT) with obliteration of a costophrenic angle on chest $\mathrm{x}$-ray as eligible for compensation. Previous studies report significant airflow limitation and functional impairment in these patient.

Methods A database search from 08/2005 to 01/2013 for DPT as agreed by a multi-disciplinary team meeting as fulfilling the criteria for a claim. Any IIDB advice given, records of those who applied, smoking history and lung function tests at presentation were interrogated. Lung function was performed by a specialist cardio-pulmonary technician by body plethysomography. Airflow obstruction defined by ARTP/BTS criteria as FEV1/ FVC <70\% and severity of airflow obstruction by FEV1 $(50-80 \%$ predicted as mild, $30-49 \%$ predicted as moderate and $<30 \%$ as severe obstruction respectively).

Results 39/50 male subjects with DPT attended follow-up.
$100 \%$ were given written advice of their eligibility to claim IIDB.

24/39 (62\%) who were followed up applied for IIDB, with disability ranging from $15-100 \% .2$ were recorded as having less than $15 \%$ disability. Of 15 who did not apply, 4 patients' exposure was not work related and 11 chose not to apply.

4/39 (10\%) were never smokers and 30/39 (77\%) exsmokers.

Lung function results were available for $43 / 50$ at presentation (table 1).

Conclusions Our group of patients had a significantly high smoking history. Airflow limitation with DPT is the predominant lung function abnormality seen. Despite their disability, patients chose not to apply for IIDB as above.

The airflow limitation may be progressive and hence important to repeat the lung function tests over a time period to see if this deteriorates further contributing to worsening disability.

\section{REFERENCES}

1. Abnormal lung function associated with asbestos disease of the pleura, lung and both; comparitive analysis, Thorax 1991

\begin{tabular}{lll} 
Abstract P208 Table 1. Lung & Function & patterns. \\
\hline Spirometry & Total & $\%$ \\
\hline Mild obstruction $\mathrm{n}=16$ & 20 & 46.5 \\
Moderate airflow limitation $\mathrm{n}=4$ & & \\
Restriction of airflow & 3 & 7 \\
Mixed pattern & 3 & 7 \\
No obstruction & 17 & 39.5 \\
Total & 43 & 100 \\
\hline
\end{tabular}

\section{Investigation and management of pleural disease}

\section{P209 THE CLINICAL UTILITY OF PLEURAL LYMPHOCYTE SUBSET ANALYSIS IN UNDIAGNOSED EFFUSIONS}

${ }^{1} \mathrm{R}$ Bhatnagar, ${ }^{1} \mathrm{AO}$ Clive, ${ }^{2} \mathrm{~N}$ Zahan-Evans, ${ }^{2} \mathrm{AJ}$ Morley, ${ }^{3} \mathrm{PF}$ Virgo, ${ }^{2} \mathrm{ARL}$ Medford, ${ }^{2} \mathrm{JE}$ Harvey, ${ }^{4} \mathrm{CE}$ Hooper, ${ }^{5} \mathrm{SH}$ Otton, ${ }^{6} \mathrm{M}$ Brett, ${ }^{1} \mathrm{NA}$ Maskell; ${ }^{1}$ University of Bristol Academic Respiratory Unit, Bristol, United Kingdom; ${ }^{2}$ North Bristol Lung Centre, North Bristol NHS Trust, Bristol, United Kingdom; ${ }^{3}$ Department of Biochemistry, North Bristol NHS Trust, Bristol, United Kingdom; ${ }^{4}$ Worcestershire Acute Hospitals NHS Trust, Worcester, United Kingdom; ${ }^{5}$ Department of Haematology, North Bristol NHS Trust, Bristol, United Kingdom; ${ }^{6}$ Department of Cellular Pathology, North Bristol NHS Trust, Bristol, United Kingdom

\subsection{6/thoraxjnl-2013-204457.361}

Introduction Blood and tissue lymphocyte subsets (LS) analysis are routinely used in the diagnosis of a number of haematological conditions. Samples cost $£ 25$ to process and are technically labour intensive. The 2010 BTS pleural guidelines suggest LS may be useful in cases of suspected lymphoma, but there is no evidence supporting their utility or position in pleural diagnostic algorithms.

Methods Using a prospectively-maintained database of all undiagnosed pleural effusions, we analysed patients presenting to our service from 2009-2011. Fluid was initially sent for cytology and cell differential. Patients with $\geq 50 \%$ fluid lymphocytes at first sampling, with no definite cytological evidence of carcinoma, and who underwent a further pleural procedure, had 